\title{
NEUROEDUCACIÓN EN DIÁLOGO: NEUROMITOS EN EL PROCESO DE ENSEÑANZA-APRENDIZAJE Y EN LA EDUCACIÓN MORAL
}

\author{
DANIEL PALLARÉS-DOMÍNGUEZ \\ Universitat Jaume I de Castellón
}

\begin{abstract}
RESUMEN: Este trabajo se plantea como una breve revisión crítica sobre algunos de los temas actuales que se están estudiando en la intersección entre neurociencia, educación y ética. El primer objetivo es reflexionar sobre la relación que mantienen supuestos básicos que definen la conceptualización actual de la neuroeducación. Manteniendo un diálogo interdisciplinar, el segundo objetivo será analizar algunos de los neuromitos en el proceso de enseñanza-aprendizaje. El tercer objetivo es descubrir ciertos neuromitos en la educación moral, especialmente a partir de lo que algunos de los autores más relevantes en neuroética han asegurado sobre el comportamiento moral en base a los estudios del cerebro humano. A través de una metodología argumentativa, se propondrá una dirección orientativa en el quehacer de esta nueva neurociencia social que sea dialógica e interdisciplinar, manteniendo siempre un compromiso ético con el pleno desarrollo del ser humano en la búsqueda de exigencias de justicia universal.
\end{abstract}

PALABRAS CLAVE: neuroeducación, interdisciplinar, neuromitos, enseñanza-aprendizaje, educación moral.

\section{Neuroeducation in dialogue: neuromyths in theaching-learning process and in moral education}

ABSTRACT: This paper presents a brief critical review of some of the current issues that are being studied in the intersection of neuroscience, education and ethics. The first objective is to reflect on the relation between the basic assumptions that define the current conceptualization of education. Keeping an interdisciplinary dialogue, the second objective is to analyse some of the neuromyths in the teachinglearning process. The third objective is to find certain neuromyths in moral education, especially from what some of the most important authors in neuroethics have established about the moral behaviour according to studies of the human brain. Through and argumentative methodology, we will propose an orientative dialogue and interdisciplinar direction in the development of this new social neuroscience, always maintaining an ethical commitment to the full development of human beings in the pursuit of universal justice demands proposed.

KEY WORDS: neuroeducation, interdisciplinary, neuromyths, learning-teaching process, moral education.

\section{UNA BREVE APROXIMACIÓN A LA NEUROEDUCACIÓN ${ }^{1}$}

La neuroeducación, entendida como la introducción del estudio neurocientífico del cerebro en los procesos de aprendizaje y su posible aportación a la enseñanza, nace como ciencia interdisciplinar a finales de los años 90 del

1 Este estudio se inserta en el Proyecto de investigación de I+D+I del Ministerio de Economía y Competitividad «Política, empresa y educación desde la neuroética aplicada» [Ref. FI2013-47136-C2-2- P]. 
s. xx. Esta década, conocida comúnmente como «década del cerebro», fue testigo del giro que protagonizó la neurociencia hacia el estudio de las ciencias sociales y humanísticas, dando lugar a otras neurociencias sociales tales como la neuroética, el neuromarketing o la neuropolítica².

El estudio de las bases o sustratos neurales de las actividades que el ser humano realiza en sociedad, fue posible gracias al desarrollo tecnológico de las neurociencias ${ }^{3}$, heredado en gran parte de la psicología cognitiva en conjunción con otras disciplinas como la biología y la psicología evolutiva. Estas técnicas, especialmente las neuroimágenes por resonancia magnética funcional (fMRI), supusieron que cada vez se le diera más importancia al «¿dónde?»cerebral que al «¿cómo?» neural, debido al gran potencial atractivo que supone la observación de una imagen cerebral (Dolan, 2008, p. 46). Así, las técnicas de neuroimagen se convirtieron en la principal herramienta para medir la actividad neural asociada con un estado de la mente o comportamiento, pero también en herramientas de control y manipulación de la aplicación tecnopráctica de los resultados alcanzados (García-Marzá, 2012).

Progresivamente la neurociencia fue adquiriendo una pertinencia y normatividad científica que, no dejando de ser criticada (Salles, 2013), se introdujo en el estudio de las ciencias sociales. La educación no fue una excepción, y lo que hoy se conoce como neuroeducación o Mind Brain Education (MBE) ${ }^{4} \mathrm{se}$ afianzó como término académico partir de las definiciones de diversos autores.

Por un lado, A. Battro y D. Cardinali la definieron desde una perspectiva más integradora y pedagógica como: «una nueva interdisciplina y transdisciplina que promueve una mayor integración de las ciencias de la educación con aquellas que se ocupan del desarrollo neurocognitivo de la persona humana» (Battro \& Cardinali, 1996, p. 1). También H. Koizumi la definición en esta línea, entendiendo la neuroeducación como una transdisciplina de los procesos de desarrollo de aprendizaje humano (Koizumi, 2008).

En ambas definiciones destaca el término «transdisciplina», que sin duda va más allá de la interdisciplinariedad científica tan renombrada en las neurociencias sociales. Es decir, no sólo representaría un trabajo conjunto entre neurocientíficos y educadores para intercambiar puntos de vista con respecto

2 Algunos han entendido en este aspecto que las neurociencias podrían dar lugar a una «neurocultura» (MoRA, 2007). Sin embargo, desde una perspectiva más prudente e interdisciplinar, en este estudio se toma como modelo el término de «neurofilosofía práctica» para hacer referencia a la relación fructífera y no colonialista entre neurociencia y ciencias sociales (CORTINA, 2012).

3 Tal y como advierte W. Glannon las más comunes y que han tenido más influencia han sido la tomografía computarizada (CT), la tomografía por emisión de positrones (PET), la imagen por resonancia magnética (MRI), la tomografía axial computarizada por fotón único (SPECT), y la imagen por resonancia magnética funcional (fMRI) (GLANNON, 2007, p. 1). Aunque hoy en día se pueden distinguir entre las más avanzadas, el brainbow o la optogenética (SAlLes \& Evers, 2014, p. 8).

4 En este texto se tomarán como sinónimos ambos términos y se utilizará sólo el de «neuroeducación». 
a un objetivo común —interdisciplina - sino que se fraguarían nuevos parámetros de investigación conjunta entre ambas disciplinas para responder a cuestiones que trascienden el nivel disciplinar en el que se encuentran y que requieren de una reflexión en los niveles fisiológico, fenomenológico, teleológi-

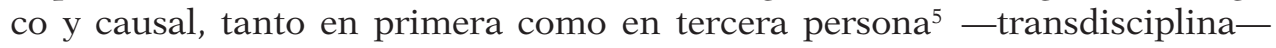
(Campbell, 2011, p. 8).

Por otro lado, desde una perspectiva más vinculada con el ámbito neurocientífico, autores como D. Ansari (2008) o R. Campbell (2011), entienden la neuroeducación como una ciencia cognitiva aplicada.

En cualquier caso, la neuroeducación pretende destacar el papel crucial del estudio del cerebro en la educación. No sólo destacarlo, sino hacerlo explícito a la hora de crear metodologías, revisar presupuestos, o estudiar trastornos del proceso de enseñanza aprendizaje (Marina, 2012). No faltaron las críticas a la imposibilidad de tal proyecto, como las de J. Bruer (1997), quien señaló tres puentes lejanos en el terreno neuroeducativo. En primero se refería al poco conocimiento de la estructura funcional del cerebro para poder unirlo a la práctica educativa. El segundo se refería a la brecha entre la educación y la psicología cognitiva ${ }^{6}$. El tercero a la distancia que existía entre la psicología cognitiva y la neurociencia ${ }^{7}$.

A pesar de estas críticas, la neuroeducación ha destacado una bidireccionalidad dialógica entre la neurociencia y la educación (Ansari, et al., 2011, pp. 3-4), en la que se pueden diferenciar cuatro niveles de relación: descriptivo, prescriptivo, de revisión y terapéutico.

El nivel descriptivo se refiere a la utilización de la tecnología neurocientífica aplicada al proceso de enseñanza aprendizaje con el objetivo de saber cómo aprende el cerebro (Fischer et al., 2007, p. 1). Los ejemplos en este nivel son muy variados. Uno sería la forma en que diferentes áreas neurales intervienen en el proceso de lectura, la identificación de personas con problemas de lectura y la forma para ayudarles con intervenciones no invasivas del cerebro (Ansari, 2008, p. 6). Otro ejemplo sería la información sobre los beneficios

5 Los problemas que se generan actualmente en el panorama educativo, tales como como los trastornos del aprendizaje, la dependencia de las nuevas tecnologías o el acoso escolar requieren de esfuerzos comprensivos y dialógicos para poder así trascender las propia disciplina a la hora de resolverlos.

6 Para algunos autores, la simple unión entre neurociencia y educación nos llevaría a una «neurociencia educativa». En la aplicación de los supuestos neurocientíficos a la práctica pedagógica es necesario un saber mediador, que algunos han identificado con la psicología cognitiva (Blackmore \& Frith, 2007, p. 4; TokUhama-Espinosa, 2011, p. 20). No obstante, en el marco en el que se sitúa este trabajo, pensamos que la mediadora entre las propuestas neurocientíficas y su aplicación al aula debería ser la ética, que es la única de velar por la aplicación tecnológica de forma que se garanticen la integridad y autonomía del ser humano.

7 Estas distancia se ha reducido considerablemente en la última década, por lo que el autor se ha visto obligado a reconsiderar su posición hacia una más optimista (BRUER, 2005), quizá en parte también debido a las respuestas y críticas recibidas (TOMMERDAHL, 2010). 
de ciertas actividades en la arquitectura neural, como la percepción visual en malabaristas o de orientación espacial en taxistas (Hall, 2005, p. 14).

El nivel prescriptivo haría referencia a cómo la pedagogía incorpora el estudio de los avances y descubrimientos del cerebro para diseñar metodologías educativas que vayan acordes con él. Los principales casos suelen darse en el estudio del lenguaje y las matemáticas ${ }^{8}$. Por ejemplo en metodologías de procesamiento semántico y aprendizaje del vocabulario (Goswami, 2004, p. 7) o las metodologías que parten del «sentido numérico» que se localiza en las áreas intraparietales, y se activan también con el procesamiento numérico arábico y las palabras que se refieren a números (Ansari, et al., 2012, pp. 109-110; Dehaene et al. 1998).

El nivel terapéutico haría referencia tanto al tratamiento de enfermedades y trastornos del aprendizaje en el ámbito clínico, como a la preocupante aplicación psicofarmacológica directa en centros escolares, a partir de productos como ritalin o adderall (Stein et al., 2011, p. 804). Aunque no es el objetivo del presente estudio, sería necesaria una reflexión sobre las condiciones éticas de justicia, así como de necesidades educativas, que justificarían una intervención instrumental directa sobre el comportamiento de los discentes, principalmente a través de psicofármacos y nootropos (Farah, 2010; Sheridan et al., 2006).

El nivel de revisión, haría referencia a la revisión neurocientífica de algunos de los presupuestos en los que se ha basado la enseñanza tradicional que con respecto al funcionamiento del cerebro y su relación con el aprendizaje (Fischer et al., 2007, p. 1). Este será el nivel que se va a tratar en profundidad en este texto en las siguientes páginas.

\section{NeURomitos EN EL PROCESO DE ENSEÑANZA-APRENDIZAJE}

La neuroeducación entiende los neuromitos generalmente como falsas ideas, creencias, interpretaciones o extrapolaciones que han trascendido a la opinión pública a pesar de haber sido desterradas o invalidadas por la neurociencia. Suelen ser explicaciones o hipótesis simples, o de carácter muy general, no demostradas pero que por su propio contenido o significado han calado muy hondo en la opinión pública en parte debido a la difusión de los medios de comunicación y que cuesta mucho desarraigar (Geake, 2008, p. 123; OECD, 2007, p. 109).

Hay que tener en cuenta que la neurociencia basa gran parte de su proceder en el trabajo de laboratorio. El origen de un neuromito se produciría cuando ese trabajo realizado en condiciones artificiales de laboratorio trasciende las

8 También podrían incluirse las metodologías que incorporan el estudio del sueño (FALLONE et al., 2002), la experiencia directa con el medio, la importancia del ejercicio físico, y los estudios sobre cronoeducación, y sobre todo la relación entre emoción y cognición en el proceso de enseñanza-aprendizaje (ANSARI et al. 2012, pp. 111-112; GosWAMI, 2004, pp. 9-11). 
paredes de dicho espacio sin ser debidamente contrastado, sin ser canalizado adecuadamente a través de los medios de comunicación, y siendo aplicado directamente al diseño institucional como en este caso a la educación (Geake, 2008, p. 124).

Su persistencia en la opinión pública residiría en la gran capacidad de seducción de la neurociencia, sobre todo a través de las neuroimágenes funcionales (Weisberg et al., 2008; McCabe \& Castel, 2008). El nacimiento de un neuromito puede ser intencionado o no, y quizá existan causas que no se contemplan aquí, como por ejemplo presiones de compañías farmacéuticas para que se receten nuevos psicofármacos, o modas de supuesta innovación educativa que se basan en hipótesis no contrastadas científicamente. En caso de que persistan, no permitirían un progreso en el proceso de enseñanza-aprendizaje. A continuación se tratarán algunos de ellos.

\subsection{El condicionamiento del aprendizaje hasta los tres años}

Uno de los neuromitos más extendidos es el que enuncia que todo lo que va a condicionar casi la totalidad del aprendizaje en la vida debe darse a la edad de tres años (Dekker et al., 2012, p. 2; Tokuhama-Espinosa, 2011; OECD, 2002, p. 111, 2007, pp. 109-110). Este neuromito tiene un origen doble.

En primer lugar, se debe a una exageración de dos condicionantes fisiológicos: la neurogénesis y las inaptogénesis. Una importante cantidad de cambios fisiológicos tienenlugarentreelnacimientoylostresaños,peroestonosignific aquesean «críticos» ni que deba exagerarse con respecto a este periodo. Cualquier aprendizaje implica, a nivel neural, la creación y el fortalecimiento o debilitamiento de sinapsis elemento fundamental de la relación entre neuronas. La sinaptogénesis y la neurogénesis son intensas en los primeros años de vida, y por eso en parte, los niños/as son más propensos a aprender a un ritmo más rápido (Huttenlocher \& Dabholkar, 1997). Lo que se podría decir es que si se estimula positivamente a los infantes en sus primeros tres años de vida, se les proporcionaría la base para que vayan fortaleciendo sus capacidades a lo largo de la vida con el aprendizaje.

En segundo lugar, se debe a que uno de los primeros focos de estudio de la neuroeducación fueron los llamados «periodos críticos del aprendizaje». Quizá el término "crítico» no sea el más adecuado en el proceso educativo. El origen etológico de este concepto ${ }^{9}$, quizá sea apropiado en el caso de los estudios con animales, pues la falta de aprendizaje en la situación de impronta puede traer

9 Uno de los primeros en utilizar este término en el ámbito educativo fue K. Lorenz. Desde una perspectiva evolutiva lo utilizó para dar nombre a un proceso que observó en sus experimentos con animales en la década de 1970. Descubrió que los polluelos en edad muy temprana quedaban permanentemente atraídos por el objeto mayor de su entorno más cercano y que seguían a todas partes, normalmente su madre. A este efecto lo denominó «imprinting» (LORENZ, 1981). 
consecuencias irreversibles ${ }^{10}$.Sin embargo, en el caso de los seres humanos es diferente, y es preferible hablar de «periodos sensibles del aprendizaje». Un ejemplo lo constituiría la lectura. Como el tiempo de organización de las funciones neurales que implica la lectura es más extenso que el de funciones más específicas - como el de la visión- los impactos ambientales pueden producir efectivamente cambios, pero éstos no serán irreversibles, es decir, serán sensibles pero no críticos (Lipina, 2014, p. 74).

\subsection{Los seres humanos usamos el $10 \%$ del cerebro}

El segundo de los neuromitos es el que hace referencia a que los seres humanos utilizamos solamente el 10\% de la capacidad cerebral (OECD, 2007, pp. 113-114; Geake, 2008, p. 127; Tokuhama-Espinosa, 2011). Su origen podría ser la entrevista que en 1920 realizó A. Einstein en la radio, cuando precisamente éste hizo referencia a que el ser humano sólo usaba el 10\% de su cerebro. Sin embargo, esto lo hizo para animar a la gente a que pensara y se formara más y mejor. Una década más tarde, en $1930 \mathrm{~K}$. Lashley intentó estudiar al cerebro humano usando shocks eléctricos. Ciertas áreas que no reaccionaban ante estos shocks, K. Lashley dijo que no tenían ninguna función, y acuñó el término de «corteza silenciosa» - silent cortex.

Otro de los posibles orígenes de este neuromito quizá se deba a la relación entre las neuronas y las células gliales. A grandes rasgos suelen haber unas diez células gliales por cada neurona. Comúnmente se entiende que las células gliales aportan nutrientes pero no transmiten impulsos nerviosos. Así, la transmisión de impulsos nerviosos, hablando en términos globales, se realizaría a través del 10\% de las células del cerebro (Rose, 2008, p. 176).

Como contrargumentación de este neuromito, se puede decir que, en realidad, el cerebro permanece totalmente activo. Ningún área permanece 100\% inactiva, incluso durante el sueño (OECD, 2007, p. 113). Además, este neuromito tampoco se justificaría desde el punto de vista evolutivo. El cerebro apenas ocupa el $2 \%$ del peso corporal, pero consume alrededor de un $20 \%$ de la energía. Seguramente nuestro propio sistema, evolutivamente hablando, no habría permitido que el $20 \%$ de la energía la consuma un órgano que desperdiciaría el 90\% de su capacidad (Geake, 2008, p. 127).

\subsection{La utilización preferente de un hemisferio condiciona el aprendizaje}

La lateralización cerebral constituye el origen de otro neuromito (Ansari, 2008, pp. 9-10; Goswami, 2004, pp. 10-11). Este neuromito entiende que

10 En la década de 1960, T. Wiesel y D. Hubel llevaron a cabo una serie de experimentos para estudiar la adaptabilidad neuronal ante un proceso de privación del sentido de la vista, en un solo ojo, en gatos durante la edad temprana. Las consecuencias fueron un deterioro grave de las conexiones neuronales en las áreas visuales cerebrales del ojo que taparon, casi quedando ciegos de ese ojo (Blackmore \& Frith, 2007, pp. 46-47; Bruer, 1997, p. 7). 
según el hemisferio que predomine, los seres humanos tenemos un tipo de pensamiento y comportamiento. De esta forma, el hemisferio izquierdo sería la sede del pensamiento más racional y analítico. Estaría relacionado con las funciones de lectura y escritura —lenguaje_-, el pensamiento matemático, la resolución de problemas, y las operaciones lógico-formales. El hemisferio derecho, en cambio, conformaría la sede de la intuición y la emoción. (OECD, 2007, p. 114). Este neuromito parece tener un doble origen.

Por un lado, podría haberse originado por A. Ladbroke, quien en 1844 publicó A New View of Insanity: Duality of the Mind, en la que describió los hemisferios cerebrales como independientes, atribuyendo a cada uno de ellos un tipo de pensamiento (OECD 2007, p. 114). Por otro lado, la obra Psychology of Consciousness (1970) de R. Ornstein, utilizó por primera vez el término «westerners ${ }^{11}$ para referirse a aquellas personas que utilizaban preferentemente el hemisferio izquierdo, y en su interpretación, eran más propensos a tener un pensamiento lógico-formal (OECD, 2007, p. 116).

Teniendo en cuenta las aportaciones de la neuroeducación, la división propuesta por la lateralización hemisférica cerebral es muy simplista, pues organiza el cerebro en dos sistemas conscientes, mientras que más bien estaría organizado en múltiples subsistemas mentales dinámicos e interactivos. Es cierto que existen ciertas tareas que requieren de una mayor implicación de un hemisferio cerebral, como el reconocimiento de rostros o la producción del lenguaje hablado, sin embargo, no hay ninguna tarea que requiera sólo de la actividad de un solo hemisferio. Pues ambos trabajan conjuntamente en cualquier tarea cognitiva (Gazzaniga, 2012, p. 59).

\subsection{Los estilos preferentes de aprendizaje (VAK)}

Los estilos de aprendizaje preferentes hacen referencia a modalidades sensoriales: visual, auditiva y kinestésica (VAK). Según estas modalidades, se realiza un test a los estudiantes para saber cuál es su estilo predominante y preferente de aprendizaje, y se les enseña de acuerdo con ello (Dunn \& Price, 1984). En esta tipología de aprendizaje se asumen implícitamente dos cosas. En primer lugar, se toma como punto de partida no sólo la modalidad sensorial, sino la modalidad sensorial «preferente» por el estudiante. En segundo lugar, entiende que las modalidades sensoriales son estructuras neurales que pueden separarse, y que el proceso de enseñanza-aprendizaje puede partir únicamente de una de ellas (Coffield, 2004).

11 Sin embargo, el término "westerners» poco tenía que ver con el lado izquierdo, sino más bien se refería a una diferencia cultural, ya que asoció implícitamente el pensamiento emocional e intuitivo con las culturas orientales y el pensamiento matemático y lógico-formal con las culturas occidentales. Esta relación de lo cerebral con lo cultural supuso el paso de asociar los hemisferios no sólo con diferentes tipos de pensamiento y aprendizaje, sino también con el comportamiento y la personalidad, dando lugar así a personas caracterizadas por el hemisferio izquierdo o el hemisferio derecho (GEAKE, 2008, p. 129). 
Estas dos premisas son potencialmente problemáticas. En cuanto a la primera, que un estudiante prefiera un estilo a otro no significa que sea efectivo, sólo que le gusta más, y las causas de ello pueden ser variadas, y diferir mucho de las que tienen que ver realmente con el aprendizaje. En cuanto a la segunda, esta teoría concibe que información sensorial diferente se procesa en estructuras cerebrales distintas. Pero no sólo diferentes, sino separadas e independientes. Esto supone volver a una concepción frenológica del cerebro. Además, esta propuesta entiende que la información aprendida mediante una modalidad sensorial se procesa a nivel neural de una forma diferente e independiente de lo que se aprende mediante otra modalidad. En este sentido, la neuroeducación ha destacado que es incorrecto asumir que sólo una modalidad de procesamiento sensorial está involucrada en cualquier tipo de aprendizaje (Dekker, et al., 2012, p. 2; Ansari, 2008, pp. 9-10; Krätzig \& Arbuthnott, 2006).

Todos los neuromitos explicados en este apartado hacen referencia a diversos aspectos del proceso de enseñanza-aprendizaje. Pero también es preciso señalar ciertos neuromitos que se seguirían de la aplicación de los descubrimientos en neuroética a la educación moral. Estos neuromitos tendrían un carácter diametralmente opuesto a los anteriores. Es decir, no sería la neurociencia —en este caso la neuroética- quien los descubriría, sino quien los implantaría si se educara moralmente teniendo en cuenta algunas de sus aportaciones, como se verá a continuación.

\section{NeURomitos EN La educación MORAL}

Teniendo en cuenta las aportaciones de algunos autores (Gazzaniga, 2012; Churchland, 2012; Haidt, 2001) los principales neuromitos que se desprenderían si se aplicaran las aportaciones neuroéticas a la educación moral serían: (I) que los seres humanos estamos fraguados a nivel neural para desarrollar una conducta que nos impulsa a estar bien con los cercanos, mantener posiciones de respeto y tolerancia para maximizar nuestro interés en la supervivencia y el bienestar; (II) que no existe la libre voluntad humana porque es el cerebro el que toma la decisión antes que nosotros, de tal forma que a lo sumo podría sólo existir una ilusión de libertad humana que nos ayudaría a convivir socialmente; (III) y que el modelo de referencia para la educación moral debe ser el del intuicionismo social y no el del desarrollo del juicio moral a través de la capacidad de dar razones —impulsado principalmente a través de L. Kohlberg ${ }^{12}$.

12 Precisamente la obra de L. Kohlberg (1976; KohlBerg et al., 1975) proporciona un marco teórico para el abordaje de las preocupaciones éticas sobre la enseñanza, un marco conceptual para facilitar a los docentes la integración de los contenidos y procesos morales en la enseñanza. Más que integrarlos — que ya de por sí están integrados- hacerlos visibles. 


\subsection{El interés por el cercano y el desinterés por el lejano}

En cuanto al primer neuromito (I), hay que destacar los estudios de la psicología y la biología evolutivas (Cela-Conde, 2005; De Waal, 2005), además de los estudios sobre endocrinología a los que hacían referencia autores como P. S. Churchland (2012), apuntaban a una dirección: la de maximizar la supervivencia y el bienestar, de forma que potenciáramos la relación con los seres cercanos y nos despreocupáramos del vínculo con los seres lejanos a nuestro círculo social.

Este tipo de conducta hace referencia a lo que A. Cortina llamó «individualismo ilustrado» (1998, pp. 59-74). Sin duda, existen connotaciones históricas que han llevado a que comúnmente se considere la noción de «individuo» no sólo como un ser organizado respecto a su especie, sino también a considerarlo como un ser que entiende su libertad como independencia respecto a los demás, y valore las relaciones sociales en la medida que le benefician (Cortina, 1998, p. 62). Pero la ética no puede justificarse sólo atendiendo a un sentimiento de simpatía. Es decir, la pregunta ¿por qué debo? no puede justificarse por un sentimiento que vaya sólo en una dirección, como la simpatía. Debe, por el contrario, poseer un camino de vuelta, una reciprocidad: «No sólo existe mi sentimiento de simpatía, existe también, aunque socialmente configurada, la exigencia legítima de otros que debo reconocer si no quiero caer en la injusticia»(Cortina, 2007, p. 97).

Desde estas perspectivas neuroéticas, se desprende que lo razonable sería la capacidad que cada individuo tenemos de maximizar nuestro propio interés personal y grupal. En el terreno moral, el interés sería suficiente para lograr una "moral por acuerdo", pero ¿realmente es eso en lo que querríamos educar moralmente? Hay que tener en cuenta que el acuerdo moral basado en el interés presenta muchas deficiencias:

1) es casi imposible diseñar unos contenidos en los que «todos ganen»; 2) quien parte de su propio interés para sellar el pacto, intentará eludirlo y dar la apariencia de que lo cumple; 3 ) la norma de que «hay que cumplir los pactos» sólo la pueden seguir quienes estén motivados moralmente para ello, es decir, aquellos para los que lo moral tiene un valor (Cortina, 1998, p. 70).

Lo que estas perspectivas neuroéticas olvidan es que el individualismo no agota el terreno moral, sino que es un paso previo para llegar a algo mucho más importante. Una verdadera racionalidad humana plena en el terreno moral se caracterizaría porque quienes la practican no sólo obran por interés, sino que toman interés en aquello que es por sí mismo valioso. Por ello, como dice A. Cortina, más allá del interés, la prudencia y la felicidad, ingresamos en el ámbito de la justicia (Cortina, 1998, p. 74). Pero si no somos capaces de apreciar lo que es valioso por sí mismo, y tan sólo lo que nos produce conveniencia, no podremos alcanzar los grandes desafíos de justicia universal. En este sentido

Su obra simboliza primariamente la revitalización y la necesidad de la educación moral (Hers et al., 1997, p. 18). 
¿para qué servirá la neuroética si sólo se propaga la creencia en que el cerebro los proporciona pautas de actuación para la supervivencia y el bienestar personal o de unos pocos allegados? ¿No sería esto más bien un retroceso en la educación moral respecto al «progreso moral» que ha costado tanto al ser humano alcanzar? (Habermas, 1981).

\subsection{La libre voluntad no existe por ser una ficción del cerebro}

El segundo de los neuromitos lo constituyen las propuestas neurocientíficas que negaban la libertad humana (II). Para ello algunos autores suelen basarse en los estudios ya clásicos del B. Libet sobre el readiness potential (RP) (Libet et al., 1983; Libet, 1999). Autores como D. Wegner (2002), F. Rubia (2009) o M. Gazzaniga (2012) y otros que trabajan en reconstrucciones más recientes del experimento de Libet (Soon et al., 2008) suelen dar tres argumentos para ello: que la libertad es una construcción del cerebro, que está por tanto causalmente determinada, y que se inicia de manera inconsciente en nuestro cerebro.

La metodología empírica de los experimentos de Libet, así como las bases teóricas que utilizó en su estudio de los correlatos neurales de la acción voluntaria, han sido ampliamente discutidos dentro del terreno neurocientífico en los últimos años (Mecacci \& Haselager, 2015; Roskies, 2010a). Desde el punto de vista filosófico, los experimentos de B. Libet poco tienen que decir sobre la libre voluntad humana, terreno en el que la filosofía moral ha entendido generalmente como una condición fundamental para actuar responsablemente y pedirnos razones de nuestros actos.

En efecto, todos estos estudios parecen pasar por alto ciertos aspectos. Primero, que la intención originaria de B. Libet no era negar la libertad humana, sino más bien demostrar que el acto voluntario se inicia antes de la experiencia consciente del propio acto como algunos advierten (Schlegel et al., 2013), o lo que es lo mismo, refutar la hipótesis contraria que mantenía su maestro J. Eccles (Gracia, 2013, pp. 576-577).

Segundo, que el autor no tenía una intención de corte determinista y materialista, sino que eso se ha debido a interpretaciones posteriores (Habermas, 2006, p. 162). Así, lo que quiso probar Libet fue que el acto voluntario se inicia antes de que lo haga su experiencia consciente. Para ello, se precisaba una medición de la aparición de los dos fenómenos más importantes de este proceso, el potencial de preparación (RP) y la experiencia consciente. Dado que la consciencia de intención de actuar (W) no coincidía con el comienzo del RP, sino que era 350-400 ms más tarde, Libet concluyó que existía una intención de actuar anterior a la consciencia de la intención de actuar (Libet, 1999, p. 51).

Pero además, añadió que existía un periodo de $150 \mathrm{~ms}$ posterior, permitiendo al control consciente afectar al resultado final del proceso volitivo (Libet, 1999, p. 51). Por lo que no cierra la puerta al control voluntario consciente que en última instancia gobierna la acción - a lo que también se suma la afirmación de la existencia del veto consciente. Así, como se ha comentado 
anteriormente, la intención precede a la misma consciencia de intención. Pero algunos autores han concluido a partir de este punto, una tesis errónea. Han deducido que, como el acto libre tiene que ser por necesidad consciente, la libre voluntad no existe porque antes de que seamos conscientes de nuestra intención de actuar, la decisión ya está tomada.

Por tanto, concluir la negación de la libertad sería una falacia, principalmente por dos razones. En primer lugar porque, tras las palabras de Libet, lo que se podría deducir sería únicamente relaciones funcionales, no causales, sino caeríamos como D. Gracia indica, en una falacia del tipo post hoc ergo propter hoc (Gracia, 2013, p. 568). En segundo lugar, hay que advertir que Libet habla de «intención de actuar», no de "elección», que son dos cosas muy distintas. En este sentido, los experimentos de B. Libet no suponen ninguna amenaza para la libre voluntad (Roskies, 2010b).

\subsection{El modelo del intuicionismo social}

El tercero de los neuromitos (III) lo constituiría el modelo del intuicionismo social de J. Haidt y su crítica al modelo de L. Kohlberg. Tal y como resume A. Cortina (2012, p. 19) las críticas de J. Haidt al modelo de L. Kohlberg se basan en el excesivo racionalismo y deductivismo a partir de un principio moral previo con el que se toma un juicio. En efecto, J. Haidt (2001) y sus colaboradores (Haidt et al., 1993) entienden que la línea iniciada por J. Piaget y L. Kohlberg sobre el razonamiento moral a partir del juicio moral no es la más adecuada para la educación moral. Por el contrario, para ellos el juicio moral es más intuitivo, automático y guiado por la emoción que por la razón. La tesis central de J. Haidt (2001), frente a L. Kohlberg, se perfila a través de su «modelo intuicionista social»:

La tesis central del modelo intuicionista social es que el juicio moral se produce por intuiciones morales rápidas y se sigue (cuando es necesario) de lentos y ex post facto razonamientos morales. Se necesitan por lo tanto, definiciones claras del juicio moral, intuición moral y razonamiento moral ${ }^{13}$ (p. 817) [Traducción propia].

Teniendo en cuenta estas palabras, tras la definición propone nuevas conceptualizaciones de juicio moral, razonamiento moral, intuición moral, etc. Para formular este modelo, se basa en los estudios sobre psicología social y evolutiva, a la vez que en estudios sobre antropología y primatología - estas dos ciencias parecen ser un tópos común en los estudios de neuroética.

En una comparativa entre los dos procesos del razonamiento moral, expone las características de ambos y justifica que resulta mejor y más

13 "The central claim of the social intutionist model is that moral judgment is caused by quick moral intuitions and is followed (when needed) by slow, ex post facto moral reasoning. Clear definitions of moral judgment, moral intuition, and moral reasoning are therefore needed» [Texto original]. 
conveniente el proceso de la intuición. Como características del proceso de razonamiento destaca que: es lento y requiere esfuerzo; es intencional y controlable; es accesible a la conciencia y visible; requiere atención, que siempre es limitada; es secuencial; responde a una manipulación simbólica en la que el pensamiento trata de preservar la verdad; es propio de los humanos - a partir de los dos años- y de algunos simios entrenados de alguna forma; no depende del contexto; y es independiente de sus bases, podría ser conducido a cualquier regla siguiendo un organismo o máquina (Haidt, 2001, p. 818).

En contraposición con este modelo, destaca las características del proceso de la intuición: es rápido y sin esfuerzo; no es intencionado sino automático; es inaccesible, sólo hay conciencia del resultado; no requiere ejercicio de la atención; es un pensamiento holista y metafórico; es común a todos los mamíferos; depende del contexto; y depende de las bases cerebrales que lo sustentan (Haidt, 2001, p. 818).

Parece ser que su modelo de intuición social para el razonamiento moral tiene como gran baza que requiere menos esfuerzo, menos tiempo, y es más automático. Al margen de juzgar si las categorías de «esfuerzo» y «tiempo» podrían ser válidas cuando se está hablando del desarrollo del juicio moral, aquí se va a plantear por qué este modelo no debería ser el que se siga en la educación moral. Y es que, como mínimo, podrían destacarse cuatro errores en el modelo intuicionista social de J. Haidt.

En primer lugar, pese a que J. Haidt anuncia que «intuición» y «razonamiento» no se corresponden con «emoción» y «razón», acaba por tratarlos de esa forma, y además separándolos. La primera justificación de por qué no debemos educar teniendo en cuenta este modelo es precisamente una justificación educativa. Y es que si no sabemos justificar el por qué y ponerlo en lenguaje para comunicarlo, no podremos enseñarlo ni transmitirlo. Pero no sólo eso, sino que además tampoco podríamos someterlo a crítica con los demás afectados por una acción. En este sentido, J. Haidt parece olvidar lo que nos recuerda D. García-Marzá, y es que, desde la dimensión cognitiva de la ética, el hecho de renunciar a la posibilidad de encontrar y de darnos razones intersubjetivamente válidas, sería renunciar a la posibilidad de alcanzar acuerdos y de denunciar la injusticia (García-Marzá, 2005, p. 242).

En segundo lugar, también J. Haidt parece olvidar que emoción y razón forman ambas parte del proceso de razonar. Siguiendo nuevamente a D. García-Marzá (2005, pp. 242-243), todo sentimiento moral posee una triple característica: «privacidad, receptividad y cognitividad». En este sentido, debido a la privacidad y receptividad, nos sentiríamos afectados en la forma en como describe J. Haidt, rápida y automática, pero no hay que olvidar la dimensión cognitiva de la que habla D. García-Marzá. Por el hecho de que nos sintamos afectados por las acciones y comportamientos de los demás, no debemos olvidar que: «es necesario introducir una tercera propiedad para los sentimientos morales que explique estas reacciones ante las expectativas y demandas cumplidas o infringidas. Reacciones que poseen, por así decirlo, una cierta cognitividad»(García-Marzá, 2005, p. 247). Por ello, como argumenta A. 
Cortina, las teorías morales que pretendan recurrir únicamente a sentimientos sociales para desentrañar las fuentes de la obligación moral, presentan grandes dificultades (Cortina, 2007, p. 93).

En tercer lugar, J. Haidt se equivoca en el orden del procedimiento. Al criticar el modelo de L. Kohlberg, J. Haidt apunta a que existe un momento de ilusión del razonamiento objetivo, o en otras palabras, que el proceso es un razonamiento post hoc. Es decir, primero tenemos la intuición y después la intentamos racionalizar. El procedimiento desde el punto de vista de D. García-Marzá sería al revés, pues apoyándose en J. Habermas, apunta: «es la pretensión de validez que acompaña a las normas la que las convierte en obligatorias, estableciendo así la fuerza de convicción racional. Los sentimientos representan las reacciones ante su cumplimiento o infracción» (García-Marzá, 2005, p. 252). En efecto, sentimientos y razones intervienen en la conducta moral pero de forma diferente a como la expone J. Haidt. No tendría sentido indignarnos o tener repulsión por la transgresión de una norma moral si antes no la hemos definido y justificado argumentativamente, proceso mediante el cual hemos expresado públicamente las razones por las que deseamos que se cumpla.

En cuarto lugar, J. Haidt se equivoca al decir que el proceso del razonamiento no depende del contexto. Principalmente porque no da en su teoría ninguna definición de contexto. ¿Qué significa que el proceso de razonamiento propuesto por teorías como la de L. Kohlberg no dependen del contexto? ¿Se refiere al contexto social, a la convivencia en sociedad? ¿Acaso quiere decir que no dependen de las normas? Se equivocaría entonces porque, como nos recuerda D. García-Marzá (2005, pp. 247-248), la dimensión cognitiva: «nos remite a la vinculación de los sentimientos con aquellos juicios con los que relacionamos nuestro comportamiento y el comportamiento de los demás, con las demandas y expectativas recogidas y transmitidas a través de las normas».

Teniendo en cuenta estas palabras, no es que el proceso del razonamiento no dependa del contexto, sino más bien al contrario, es totalmente dependiente del contexto. El paso de un nivel a otro en la teoría de L. Kohlberg depende de la ampliación del contexto, entendido como la consideración cualitativa y cuantitativa de personas que entran en ese espacio, es decir, en la asunción de roles. En una moral preconvencional, la realidad del niño queda definida por las percepciones y deseos inmediatos que la definen para él. Si quiere salir de su postura egocéntrica, el niño debe adoptar un "principio de perspectiva», en la que amplía el contexto de consideración ${ }^{14}$ de los demás (Hersh et al., 1997, p. 49).

14 Pero la consideración de los demás, o la perspectiva social, ha de tenerse en cuenta como advierte J. Gibbs, en el pleno sentido de la palabra. Es decir, no simplemente tomando la perspectiva de otro - empatía se podría decir - sino teniendo en cuenta sus preferencias, creencias, y actitudes en lo que M. Hoffman llamó las condiciones de vida del otro (GiBbs, 2003, p. 3). 
En definitiva, el proceso del razonamiento moral no puede recaer solamente en la intuición. Si las intuiciones nos sobrevienen y nos sentimos afectados por ellas, significa que no somos totalmente dueños de ellas, y por lo tanto no podríamos ser responsables de ellas. No tendría sentido actuar solamente por ellas y a que no podríamos justificarlas y consecuentemente, tampoco enseñarlas. En un modelo de educación moral al que podría contribuir significativamente la neuroética, no se debe olvidar que: «apoyar el punto de vista moral exclusivamente en los sentimientos supone romper la relación entre voluntad y razón práctica que caracteriza el ámbito moral» (García-Marzá, 2005, p. 244). Por supuesto hay que tener en cuenta sentimientos e intuiciones en la educación moral, pero sin olvidar la dimensión cognitiva de la justificación de las acciones, pues es el único camino para llegar a las exigencias de justicia.

El modelo propuesto por J. Haidt parece muy deficiente de cara a una educación moral que, teniendo en cuenta las aportaciones de la neuroética, nos pueda llevar a formular dialógicamente exigencias de justicia universal. El modelo de L. Kohlberg sí que permite esto, justamente por la posibilidad de alcanzar un nivel postconvencional, y poder actuar en justificación a unos principios éticos universales de igualdad y dignidad como derechos fundamentales de los seres humanos (Hersh et al., 1997, p. 56).

\section{Conclusiones}

Teniendo en cuenta lo expuesto anteriormente, la neuroeducación en su ámbito de revisión de los principales presupuestos del proceso de enseñanzaaprendizaje, ha destacado una serie de neuromitos. Estos neuromitos hacen referencia a ciertas concepciones del cerebro en la práctica de la enseñanza que sería mejor abandonar para orientar mejor el proceso educativo. En este sentido, las teorías de la neuroeducación vendrían a orientar mejor la enseñanza de acuerdo con los descubrimientos del cerebro.

En la educación moral por el contrario, algunos de los descubrimientos de la neuroética podrían no contribuir positivamente al progreso moral que busca exigencias de justicia universal. En este sentido, existirían principalmente tres neuromitos: (I) que los principales dictados morales que se siguen de nuestro cerebro de forma natural serían maximizar nuestra supervivencia y bienestar en la sociedad, por lo que es preciso mantener buenas relaciones con los que nos rodean y pueden contribuir a ello, mientras que no importa tanto la relación con aquellos lejanos, de los cuales es más aconsejable desentenderse; (II) que el ser humano no posee libertad porque está completamente determinada por su cerebro, y que a lo sumo lo que puede es tener una ilusión de libertad que le permita de alguna forma convivir socialmente y crear instituciones sociales; (III) que las personas tienen intuiciones morales que les sobrevienen rápida y automáticamente. Al intentar justificarlas, lo hacen a post hoc a partir de modelos de racionalidad moral previos. De esta forma 
caen presas de una ilusión. Lo mejor sería apelar a las intuiciones, los sentimientos y la persuasión para convencer de un punto de vista moral a otra persona.

¿Realmente es este el modelo de educación moral en el que queremos educar? Un modelo de educación moral basado en estos neuromitos llevaría a la heteronomía moral cerebral, a la creencia en la ausencia o ilusión de libertad, a los juicios intuitivos emocionales para justificar nuestras acciones, y a maximizar la supervivencia y el bienestar personales, imposibilitando cualquier exigencia de justicia universal. Desde la perspectiva de este estudio, creemos que la neuroética y la neuroeducación son complementarias, y que pueden contribuir positivamente a una educación moral que vaya más allá de un nivel convencional. Para ello, se hará imprescindible educar moralmente desterrando estos neuromitos.

\section{Bibliografía}

ANSARI, D. (2008). «The brain goes to school: Strehgthening the education-neuroscience connection». Education Canada, 48(4), 6-10.

Ansari, D., Сoch, D., \& De Smedt, B. (2011). "Connecting Education and Cognitive Neuroscience: Where will the journey take us?». Educational Philosophy and Theory, 43(1), 37-42.

Ansari, D., De Smedt, B., \& Grabner, R. (2012). «Neuroeducation: a critical overview of an emerging field». Neuroethics, 5(2), 105-117.

Battro, A. M., \& Cardinali, D. P. (1996). Más cerebro en la educación. Buenos Aires: La Nación.

Blackmore, S. J., \& Frith, U. (2007). Cómo aprende el cerebro: las claves para la educación. Barcelona: Ariel.

Bruer, J.T.(1997). «Education and the Brain: A Bridge Too Far». Educational Researcher, 26(8), 4-16.

Bruer, J. T. (2005). Building bridges in neuroeducation. En Battro, A. M., Fischer, K. W., \& LÉNA, P. (Eds). Mind, brain and education (pp. 43-58). Cambridge: Cambridge University Press.

CAmpBell, S. R. (2011). "Educational Neuroscience: Motivations, methodology and implications». Educational Philosophy and Theory, 43(1), 7-16.

Cela-Conde, C. (2005). Did Evolution Fix Human Values? En Changeux, J-P., Damasio, A. R., Singer, W., \& Christen, Y. (Eds.) Neurobiology of Human Values (pp. 11-16). Berlin: Springer Verlag.

Coffield, F., Moseley, D., Hall, E., \& Ecclestone, K. (2004). Learning styles and pedagogy in post-16 learning. A systematic and critical review. London: Learning and Skills Research Centre.

Cortina, A. (1998). Hasta un pueblo de demonios: ética pública y sociedad. Madrid: Taurus.

CortinA, A. (2007). Ética de la razón cordial. Educar en la ciudadanía del s. XXI. Oviedo: Nobel.

Cortina, A. (2012). "Neuroética: presente y futuro». En Cortina A. (Ed.), Guía Comares de Neurofilosofía Práctica (pp. 9-37). Granada: Comares. 
De WaAl, F. (2005). «Homo homini lupus? Morality, the Social Instincts, and our Fellow Primates». In Changeux, J-P., Damasio, A. R., Singer, W., \& Christen, Y. (Eds.) Neurobiology of Human Values (pp. 17-36). Berlin: Springer Verlag.

Dehaene, S., Dehaene-Lambertz, G., \& Cohen, L. (1998). "Abstract representations of numbers in the animal and human brain». Trends in Neuroscience, 21(8), 355-611.

Dekker, S., Lee, N., Howard-Jones, P., \& Jolles, J. (2012). Neuromyths in education: Prevalence and predictors of misconceptions among teachers. Frontiers in Psycho$\log y, 3,1-8$.

Dolan, R. J. (2008). «Neuroimaging of Cognition: Past, Present, and Future». Neuron, 60, 496-502.

DunN, R., \& Price, G. E. (1984). Learning Style inventory. Lawrence: KS Price System.

Fallone, G., Owens, J. A., \& Deane, J. (2002). «Sleepiness in children and adolescents: clinical implications». Sleep Med Rev, 6 (4), 287-306.

FARAH, M. J. (2010). «Mind, brain and education in socioeconomic context». En FERrari, M., \& Vuletic, L. (Eds.). The Developmental Interplay of Mind, Brain, and Education (pp. 243-256). New York: Springer,

Fischer, K. W., Daniel, B. D., Immordino-Yang, H., Stern, E., Battro, A., \& Koizumi, H. (2007). "Why Mind, Brain, and Education? Why Now?». En Mind, Brain and Education, 1(1), 1-2.

García-Marzá, D. (2005). “¿Sentimientos virtuosos? El papel de los sentimientos en la vida moral». Diálogo Filosófico, 62, 241-256.

GARCÍA-MARZÁ, D. (2012). "Neuropolítica: una mirada crítica sobre el neuropoder». En Cortina, A. (Ed.), Guía Comares de Neurofilosofía Práctica (pp. 77-96). Granada: Comares.

GazZANiga, M. S. (2012). ¿Quién manda aquí? El libre albedrío y la ciencia del cerebro. Barcelona: Paidós.

GEAKE, J. G. (2008). «Neuromythologies in education». Educational Research, 50(2), 123133.

GiBBs, J. C. (2003). Moral Development and Reality. Beyond the Theories of Kohlberg and Hoffman. California: Sages.

Glannon, W. (2007). Bioethics and the Brain. Oxford: Oxford University Press.

Goswami, U. (2004). «Neuroscience and education». British Journal of Educational Psychology, 74, 1-14.

Gracia, D. (2013). «La insobornable libertad». En López-Frías, F. J. (et al.) (Eds.). Bioética, Neuroética, Libertad y Justicia (pp. 551-579). Valencia: Comares.

Habermas, J. (1981). La reconstrucción del materialismo histórico. Madrid: Taurus.

Habermas, J. (2006). Entre naturalismo y religión. Barcelona: Paidós.

Haidt, J., Koller, S. H., \& Dias, M. G. (1993). "Affect, Culture, and Morality, or Is It Wrong to Eat Your Dog?». Journal of Personality and Social Psychology, 65(4), 613-628.

Haidt, J. (2001). «The Emotional Dog and Its Rational Tail». Psychological Review, 108(4), 814-834.

Hall, J. (2005). Neuroscience and Education. A Review of the contribution of brain science to teaching and learning. Glasgow: The Scottish Council for Research in Education.

Hersh, R., Reimer, J., \& Paolitto, D. (1997). El crecimiento moral. De Piaget a Kohlberg. Madrid: Narcea.

Huttenlocher, P. R., \& Dabholkar, A. S. (1997). «Regional differences in synaptogenesis in human cerebral cortex». Journal of Comparative Neurology, 387, 167-178. 
Kohlberg, L. (1976). «Moral stages and moralisation: The cognitive-developmental approach». En Lickona, T. (Ed.), Moral development and behavior: Theory, research and social issues (pp. 31-35). New York: Holt, Rinehart and Wilson.

Kohlberg, L., Colby, A., Fenton, E., Speicher-Dubin, B., \& Lieberman, M. (1975). Collected Papers on moral development and moral education. Cambridge: Moral Education and Research Foundation.

Koizumi, H. (2008). "Developing the Brain: A functional-imaging based approach to learning and educational sciences». En Battro, A. M., Fischer K. W., \& Léna, P. J. (Eds.), The educated brain. Essays in neuroeducation (pp. 166-180). Cambridge: Cambridge University Press.

KRÄtZig, G., \& Arbuthnott, K. (2006). «Perceptual learning style and learning proficency: A test of the hyphotesis». Journal of Education Psychology, 98, 238-246.

LiBET, B. (1999). «Do We Have Free Will». Journal of Consciousness Studies, 6(8-9), 4757.

Libet, B., Gleason, C. A., Wright, E., \& Pearl, D. (1983). «Time of conscious intention to act in relation to onset of cerebral activity (readiness-potential). The unconscious initiation of a freely voluntary act». Brain, 106, 623-642.

Lipina, S. J. (2014). «Consideraciones neuroéticas de la pobreza infantil». En SALLeS, A., \& Evers, K. (Coord.), La vida social del cerebro (pp. 67-101). México D. F.: Fontamara.

LoREnZ, K. (1981). The Foundations of Ethology. New York: Springer-Verlag.

Marina, J. A. (2012). «El diálogo entre Neurociencia y Educación». Participación Educativa.Númeroextraordinario: La investigación sobre el cerebro y la mejora de la educación, 1, 7-13.

McCabe, D. P., \& CAStel, A. D. (2008). «Seeing is believing: The effect of brain images on judgments of scientific reasoning». Cognition, 107, 343-352.

Mecacci, G., \& Haselager, P. (2015). «A Reason To Be Free. Operationalizing 'Free Action'». Neuroethics, 8, 327-334.

Mora, F. (2007). Neurocultura. Una cultura basada en el cerebro. Madrid: Alianza. OECD (2007). Understanding the Brain. The birth of a learning science. Paris: OECD. Recuperado de http://www.oecd.org/site/educeri21st/40554190.pdf [Consultado el 3 de marzo de 2015].

Rose, S. P. (2008). Tu cerebro mañana: cómo será la mente del futuro. Barcelona: Paidós.

Roskies, A. (2010a). "How does neuroscience affect our conception of volition?». Annual Review of Neuroscience, 33, 109-130.

Roskies, A. (2010b). "Why Libet's studies don't pose a threat to free will». En SinnotAmstrong, W., \& Nadel, L. (Eds.), Conscious will and responsibility (pp. 11-22). New York: Oxford University Press.

Rubia, F. J. (2009). El fantasma de la libertad. Datos de la revolución neurocientífica. Barcelona: Crítica.

SAlLES, A. (2013). On the normative implications of social neuroscience». Recerca. Revista de Pensament i Anàlisi, 13, 29-42.

Salles, A., \& Evers, K. (2014). Introducción. En Salles, A. \& Evers, K. (Coord.), La vida social del cerebro (pp. 7-16). México D. F.: Fontamara.

Sheridan, K., Zinchenko, E., \& Gardner, H. (2006). "Neuroethics in education». En Illes, J. (Ed.), Neuroethics: Defining The Issues in Theory, Practice and Policy (pp. 265-276). Oxford: Oxford University Press.

Soon, C. S., Brass, M., Heinze, H-J., \& Haynes, J-D. (2008). «Unconscious determinants of free decisions in the human brain». Nature Neuroscience, 11, 543-545. 
Stein, Z., Della Chiesa, C., \& Fischer, K. W. (2011). «Ethical issues in educational Neuroscience: raising children in a brave new world». En Illes, J. \& SAHAKIan, B. J. (Eds.), The Oxford Handbook of Neuroethics (pp. 803-822). Oxford: Oxford University Press.

Tokuhama-Espinosa, T. N. (2011). Mind, Brain and Education Science. A comprehensive guide to the new brain-based teaching. New York: Norton \& Company.

Tommerdahl, J. (2010). A model for bridging the gap between neuroscience and education. Oxford Review of Education, 36 (1), 97-109.

Wegner, D. M. (2002). The Illusion of Conscious Will. Cambridge MA: MIT Press.

Weisberg, D. A., Keil, C., Goodstein, J., Rawson, E., \& Gray, J. (2008). «The Seductive Allure of Neuroscience Explanations». Journal of Cognitive Neuroscience, 20(3), 470477.

Universitat Jaume I,

Avda. Sos Baynat, s/n, E-12071 Castellón

dpallare@uji.es

Daniel Pallarés-Domínguez

[Artículo aprobado para publicación en octubre de 2016]. 